\title{
Human papillomavirus-related oropharyngeal squamous cell carcinoma: a new context for dysphagia rehabilitation
}

\author{
Julie A. Theurer $\cdot$ John Yoo $\cdot$ Anthony C. Nichols
}

Published online: 27 September 2014

(C) Springer Science + Business Media New York 2014

\begin{abstract}
Head and neck squamous cell carcinoma (HNSCC)' references a collection of malignant lesions located across several sites within the upper aerodigestive tract. Traditionally, the majority of HNSCCs arise within the oral cavity and larynx, resulting from the field cancerization effects of tobacco and alcohol exposure. However, over the last three decades, the incidence of cancers in the oropharynx has risen dramatically. Compelling evidence now links the epidemic of oropharyngeal squamous cell carcinomas (OPSCCs) to human papillomavirus (HPV) infection. This virally mediated disease boasts unique epidemiologic, mechanistic, and clinic-demographic features, of which clinicians and clinician-scientists should be aware, particularly in light of the estimated increasing prevalence of this disease in the foreseeable future. While survival and oncologic outcomes associated with HPVrelated OPSCC are very promising, what remains less clear is patient functioning following curative-intent therapeutic regimens designed relative to tobacco- and alcohol-related disease.
\end{abstract}

Keywords Head and neck cancer - Oropharyngeal squamous cell carcinoma - Human papillomavirus . Swallowing $\cdot$ Dysphagia

\footnotetext{
J. A. Theurer $(\square) \cdot$ J. Yoo · A. C. Nichols

Department of Otolaryngology - Head and Neck Surgery, London Health Sciences Centre, Schulich School of Medicine and Dentistry, 800 Commissioners Road East, London, ON N6A 4G5, Canada

e-mail: jtheurer@uwo.ca

J. Yoo

e-mail: John.Yoo@lhsc.on.ca

A. C. Nichols

e-mail: Anthony.Nichols@lhsc.on.ca
}

\section{Introduction}

Head and neck squamous cell carcinomas (HNSCCs) represent the 6th most common cancer worldwide, with approximately 500,000 new cases globally per year [1] High-risk factors for the development of these cancers continue to include exposure to known carcinogens, such as tobacco and alcohol.

Arising from anatomic sites within the upper aerodigestive tract (e.g., oral cavity, larynx, or pharynx), both the disease and its treatment can (i) significantly impair critical human functions, such as breathing, swallowing, speech, and voice production, (ii) drastically alter one's appearance, and (iii) ultimately affect one's quality of life (QOL).

Curative treatment options for HNSCC comprise surgery, radiotherapy, and biotherapies (i.e., chemotherapy, and molecularly targeted drugs). Treatment choice is affected by disease factors (e.g., TNM staging) as well as patient (e.g., age, comorbidities), and practice preferences at treatment facilities. The historical treatment philosophy for all human malignancies embraced surgical removal of the lesion to allow for further pathological staging analysis based on the availability of tissue, as well as good local disease control through wide excision [2, 3]. However, surgical access for tumors in the oropharynx necessitated an open transmandibular or transpharyngeal approach to ensure sufficient visualization of the tumor and clear surgical margins [2, 3]. The associated disruption of anatomical structures integral to respiration, speech, and swallowing often led to deleterious functional consequences, with a significant reduction in patient QOL [3, 4]. The introduction of organ preservation treatment approaches (i.e., radiotherapy, with or without chemotherapy) resulted in a major treatment paradigm shift, moving away from surgical intervention in attempts to maintain 
anatomical structures integral to the function of the speech and swallowing mechanisms [5]. First utilized as treatment for laryngeal cancer, chemoradiotherapy was associated with survival comparable to open surgery, and acceptable rates of larynx preservation [5]. The enthusiasm for organ preservation quickly led to application of radiotherapy as primary definitive treatment to other sites of the head and neck, including the oropharynx.

Management of oropharyngeal squamous cell carcinoma (OPSCC) with primary radiotherapy (with or without neck dissection) provided similar survival compared to traditional, open surgical methods with 5-year survival rates of approximately $50 \%$ for both treatment approaches [3] Moreover, the adoption of radiotherapy as an organ preservation approach significantly reduced acute and late severe treatment-related complications, including mortality compared with primary open surgery $[3,6]$.

Attempts to improve the unsatisfactory 5-year survival rates have since resulted in intensification of organ preservation approaches through implementation of altered fractionation in radiotherapy, and the addition of cytotoxic concurrent chemotherapy to radiation (CRT). These increasingly intense treatment regimens do confer a survival benefit [7-9] albeit at significant cost to the patient. The addition of concurrent chemotherapy to RT significantly increases the frequency and severity of acute and late toxicities that can drastically alter patient functioning, such as mucositis, dysphagia, xerostomia, fibrosis, osteoradionecrosis, neurotoxicity, and ototoxicity [10-12].

Against the backdrop of intensifying organ-preserving approaches, it became evident that patients with oropharyngeal cancers fared significantly better than those with other tumor sites $[9,13]$. By the beginning of the twentyfirst century, the burden of evidence pointed quite clearly to an emergent etiologic role of high-risk human papillomavirus (HPV) types (e.g., HPV16, HPV18, HPV35) in a subset of HNSCCs, almost exclusively in oropharyngeal tumors. HPV-related HNSCC is now recognized as a distinct entity with its own epidemiologic, mechanistic, prognostic, and clinic-demographic characteristics $[14,15]$. The purpose of this article is to (1) provide a primer on the disease characteristics unique to HPV-related OPSCC, (2) summarize treatment-related swallowing outcomes and dysphagia management, and (3) introduce preliminary data regarding the specific impact of HPV-positive disease on swallowing.

\section{Epidemiologic, mechanistic, clinico-demographic, and prognostic differences between HPV-negative and HPV-positive OPSCC}

Over the last three decades, the incidence of HPV-related OPSCCs has exploded, with a staggering increase of
$225 \%$ [16]. Data from a variety of geographic regions reveal the same trend of increasing incidence [17-20], solidifying that HPV-positive OPSCC is a global epidemic. Interestingly, the incidence of HPV-negative OPSCC is decreasing, with a $50 \%$ decline in incidence over the same time period [16]. The dramatic change in incidences reported for both HPV-positive and HPV-negative disease may largely be driven by behavioral factors. Decreasing rates of HPV-negative OPSCC have been related to parallel declines in smoking practices [21], resulting from successful public health campaigns [21, 22]. Reasons for the increasing rates of HPV-positive OPSCC are less well understood. It is supposed that changes in sexual practices evolved simultaneously with declining tobacco use, and thus resulted in increased exposure to sexually transmitted viruses, such as HPV [16, 23]. Indeed, infection by the sexually transmitted HPV is significantly associated with HPV-positive OPSCC, and precedes the development of malignancy [24].

$\mathrm{HPV}$ is detected in approximately $20-25 \%$ of all HNSCC mucosal lesions [14, 18, 25]. However, HPVpositive tumors are most prevalent in the oropharynx, with 50-75\% of OPSCCs reported to be HPV-positive [14, 20, 26, 27]. Low-risk types of HPV (e.g., HPV6, HPV11) are associated with the development of benign lesions, such as warts or papillomas; high-risk types, such as HPV16 and HPV18, have the propensity to be oncogenic, with a causative role implicated in mucosal cancers of the urogenital tract, and upper aerodigestive tract [15]. In HPVpositive OPSCCs, the most predominant high-risk HPV type is HPV16, occurring in approximately $87-95 \%$ of cases [14, 27, 28]. Within the oropharynx, HPV-positive cancers preferentially arise from the palatine tonsils or base of tongue (i.e., lingual tonsils) compared to other oropharyngeal sites [14, 25, 29].

\section{Mechanism of carcinogenesis}

In the majority of human cancers, tumorigenesis is in part attributable to mutation or degradation of the TP53 and RB1 genes following exposure to carcinogens, radiation, or viruses [15, 30]. These mutations degrade or disrupt the pathways of tumor suppressor proteins p53 and pRB, respectively, and thus, result in uncontrolled cell growth. In HPV-negative HNSCCs, tobacco and alcohol exposures lead to mutations of TP53 (among other genetic mutations), and thus degradation of the p53 pathway [15]. Indeed, approximately $80 \%$ of HPV-negative OPSCCs have TP53 mutations, whereas in HPV-positive HNSCC, TP53 mutations are rare [31, 32]. In HPV-related disease, viral oncoproteins (E6 and E7) lead to degradation of p53 and $\mathrm{pRB}$, respectively, leading to unsuppressed tumor growth [15]. The presence of HPV infection (specifically, E6 and 
E7 oncoproteins) appears to substitute for the TP53 mutations observed in the tumorigenic pathway of HPVnegative disease. Consequently, HPV-positive tumors demonstrate significantly fewer mutations than HPV-negative tumors [31, 32], with a reduction in mutation rate of approximately $50 \%$ in HPV-positive HNSCC [32].

\section{Clinico-demographic features}

Risk factors for HPV-positive OPSCC include demographic factors, sexual practices, and environmental exposures. HPV-positive disease occurs more frequently in younger, healthier individuals [20, 26, 33-35], and those of higher socioeconomic status $[34,36]$. At presentation, HPV-positive tumors are of earlier T-stage, and more likely to have extensive, often cystic, nodal metastases, and are thus almost always staged as advanced cancers [26].

Sexual behaviors are strong predictors of HPV-positive OPSCC [25, 27, 34]. Risk factors for oral HPV infection and HPV-positive OPSCC include: lifetime number of vaginal-sex partners, lifetime number of oral-sex partners, engagement in casual sex, early age at sexual debut, oral HPV infection, and history of genital warts [25, 27, 34]. These findings solidify the causal relationship between the sexually transmitted viral carcinogen and HPV-positive OPSCC, and implicate that development of HPV-positive disease through casual, nonsexual contact is unexpected [37].

The relationship between HPV status and other environmental exposures is less clear. Most reports indicate HPV-positive OPSCCs occur more frequently in patients who have never smoked or have had much less tobacco exposure, and with lower rates of alcohol consumption than HPV-negative OPSCC $[14,38]$. However, in the setting of HPV-positive disease, higher exposure to tobacco and alcohol negatively impacts oncologic outcomes with significantly poorer survival $[26,36]$.

Prognosis and survival

Patients with HPV-positive tumors have significantly more favorable prognosis than patients with HPV-negative HNSCC [14, 19, 20, 26, 38-43], with 3-year survival rates of 82 versus $57 \%$ in HPV-negative OPSCC [26], and an associated reduction in risk of death and cancer-specific death of approximately $60-70$ and $50 \%$, respectively [14, 20, 26]. In spite of excellent locoregional control rates, patients with HPV-positive disease experience distant metastases at a rate similar to their HPV-negative cohort [26, 36, 44], although HPV-related distant disease is disseminated in an atypical pattern, across multiple organs and unusual sites [44]. Patients with HPV-positive OPSCC tend to succumb to distant disease.
Although HPV positivity is typically indicative of better overall survival, the advantage can be obfuscated through heavy tobacco or alcohol use. Risk of death in OPSCC can be categorized as 'low risk,' 'intermediate risk,' or 'high risk' based on the factors of HPV status, pack-years of tobacco smoking, tumor stage, and nodal stage [26]. Patients deemed to be at low risk for death in OPSCC are likely to be nonsmokers with HPV-positive tumors. Patients at intermediate risk are likely to be either (1) smokers with high nodal disease and HPV positivity, or (2) nonsmokers with T2 or T3 HPV-negative tumors. Finally, patients at high risk for death are likely to be heavy smokers with large HPV-negative tumors [26]. A recent study by Broglie et al. [45] developed a synonymous risk stratification model related to alcohol use, which shows modulation in survival benefit as well, independent of smoking status.

The specific mechanism behind the HPV-associated survival advantage has not been fully elucidated. The vastly different etiologic underpinnings of HPV-positive disease, and the lack of field cancerization associated with repeated tobacco and alcohol exposure may help explain the survival advantage. In addition, HPV-positive disease appears to exhibit intrinsic radiosensitivity, allowing for greater locoregional control through treatment approaches that include radiotherapy [26, 39].

\section{Cancer treatment in the era of HPV-positive OPSCC}

Following the shift in practice toward organ preservation, standard definitive treatment for OPSCC is radiotherapy, often with concurrent chemotherapy as these tumors tend to present as advanced-stage disease, with adverse features that portend poorer survival. While this treatment confers an oncologic advantage [7-9], the high rates of acute and late treatment-related toxicities, such as hearing loss, nephrotoxicity, neurotoxicity, mucositis, xerostomia, and dysphagia [10-12], are unsatisfactory. These negative treatment sequelae translate into decreased functioning for patients, most notably impacting swallowing function [11, 12], and in turn, QOL [46]. In light of the HPV-driven escalation of OPSCC incidence, clinicians are treating a distinct cohort of patients who are known to be younger and healthier, and are more likely to be in the midst of their working and productive years than the traditional HNSCC patients [36]. With this cohort in mind, treatment philosophies are now shifting from treatment escalation focused exclusively on maximizing cure, toward the concept of treatment de-intensification in attempts to decrease the burden of toxicities while maintaining oncologic outcomes [47].

One de-escalation strategy includes the use of monoclonal antibodies $(\mathrm{mAb})$ to target specific tumor antigens in 
lieu of cytotoxic chemotherapy [48]. The addition of cetuximab ( $\mathrm{mAb}$ targeting epidermal growth factor receptor) to RT appears to be less toxic than concomitant cisplatinbased CRT [49]. Examination of oncologic and functional outcomes following RT plus cetuximab versus cisplatinbased CRT specifically in HPV-positive disease is currently underway [50], and the results are eagerly awaited. Candidacy for treatment with RT alone based on factors of tobacco use and extent of nodal disease has been proposed as an additional de-escalation strategy [47]. In the context of 'high-risk' disease (i.e., heavy smoking history and/or advanced nodal disease) [26], de-escalation is unlikely. However, for low-risk OPSCC (i.e., minimal to no smoking history and/or early or no nodal disease), RT alone may lead to similar oncologic outcomes while sparing patients chemotherapy-associated morbidities [36, 47]. Further examination of this de-escalation strategy is needed. Finally, minimally invasive surgical techniques for management of OPSCC are becoming widely accepted. Transoral laser microsurgery (TLM) and transoral robotic surgery (TORS) may provide alternate methods of de-intensifying treatment for patients with HPV-positive OPSCC. Early oncologic, functional, and QOL data for these surgical approaches are encouraging $[2,23,51-53]$. In the case of TORS, preliminary data describe lower gastrostomy tube (g-tube) rates, and the avoidance of chemotherapy in a portion of patients $[2,23$, 51]. Critical examination of TLM and TORS compared to standard treatment must now occur. The Canadian ORATOR trial (Oropharyngeal cancer Radiation versus TORS; NCT01590355) is the first randomized clinical trial designed to compare functional outcomes and survival between TORS and RT [54], in which patients are stratified by HPV status. In the United States, RTOG 1221 (NCT01953952) [55] is poised to address the same question relative to HPV-negative disease.

\section{Swallowing and dysphagia following treatment of OPSCC}

Given the relatively recent discovery of HPV as a distinct etiologic factor for OPSCC, the inclusion of HPV status as a stratifying variable in clinical trials, or as a predictive factor in data analysis, has lagged. Moreover, clinical data regarding HPV status of tumors has not been sought or recorded routinely, thus hampering retrospective study as well. As such, our understanding of the implications of HPV positivity on disease characteristics, and particularly on associated functional outcome, is in its infancy.

Several excellent recent reviews have captured the physiologic swallowing impairments and functional outcomes that commonly result following standard treatment regimens (i.e., CRT), as well as de-intensified therapies (e.g., IMRT and TORS) for OPSCC, and HNSCC in general $[51,56 \bullet, 57-59]$. The treatment literature reports a myriad of physiologic alterations that ultimately influence swallowing safety and efficiency. Frequent deficits include: decreased hyolaryngeal elevation, decreased base-of-tongue to posterior-pharyngeal-wall retraction, reduced pharyngeal contraction, reduced epiglottic inversion, and impaired laryngeal vestibule closure [56•*]. Other notable physiologic impairments such as reduced oral tongue strength and range of motion, dysfunction in upper esophageal sphincter opening, delayed triggering of pharyngeal swallow or disco-ordination $[56 \bullet \bullet, 60,61]$ occur with less frequency, but are no less detrimental to swallowing function. Penetration and aspiration are reported with great frequency, but also high variability, ranging from 7 to $95.9 \%$ and 0 to $100 \%$, respectively [56*0]. Pneumonia rate following CRT for laryngeal and pharyngeal cancers is $15 \%$ in population-based data [57]. However, the pneumonia rate is approximately $30 \%$ for patients known to have dysphagia post-treatment [11, 62], and rises to $86 \%$ among patients who develop late radiation-associated dysphagia [63]. Recovery of physiologic function post-treatment is highly variable, and may depend on the specific treatment regimen under study. In general, a significantly increased level of impairment is noted during and immediately post-CRT, with many deficits improving by 3 months post-treatment, but little change beyond that period of time [56••, 64].

The impact of TORS/TLM on swallowing physiology has yet to be fully explored. Instrumental assessment has not been utilized to describe the physiologic profile of the swallowing mechanism post-surgery. With the majority of TORS/TLM patients also undergoing adjuvant therapy [51], it would be beneficial to appreciate physiologic impact attributable solely to surgical intervention that may be present prior to the addition of the physiologic effects of (chemo)radiotherapy.

The functional implications of the myriad swallowing impairments have been captured as treatment toxicities, changes in diet/oral intake, and feeding tube dependency. Within the context of clinical trials, impact of treatment on swallowing function is typically captured by documentation of 'dysphagia toxicity' (see Table 1) through the Common Terminology Criteria for Adverse Events [65], a 5-point scale used to document all treatment-related toxicities or side effects [66]. The CTCAE codes can be used to capture both acute ( $<90$ days post-treatment) and chronic ( $>90$ days post-treatment) toxicities, and in this way, is often used to document acute dysphagia and long-standing swallowing difficulty resulting from treatment. Following CRT, acute grade $\geq 2$ dysphagia and acute grade $\geq 3$ dysphagia occur in $6-93 \%$ and $7-65 \%$ of trial patients, respectively [67-70]. Late grade $\geq 2$ dysphagia and grade $\geq 3$ dysphagia occur in 
Table 1 CTCAE v.4.03 [65] toxicity grades for documenting swallowing difficulty as a treatment-related adverse event

\begin{tabular}{ll}
\hline $\begin{array}{l}\text { Dysphagia toxicity } \\
\text { grades }\end{array}$ & Description \\
\hline Grade 1 & $\begin{array}{l}\text { Symptomatic, able to eat regular diet } \\
\text { Grade 2 }\end{array}$ \\
Grade 3 & $\begin{array}{l}\text { Severely altered eating/swallowing; tube } \\
\text { feeding or TPN or hospitalization indicated }\end{array}$ \\
Grade 4 & $\begin{array}{l}\text { Life-threatening consequences; urgent } \\
\text { intervention indicated }\end{array}$ \\
Grade 5 & Death \\
\hline
\end{tabular}

$2-36 \%$ and $0-13 \%$, respectively [67-71]. When functional swallowing outcome is characterized as the need for g-tube, 29-62\% of patients require enteral support during CRT [62, $72,73]$, with rates dropping to $9-23 \%$ at 6 months posttreatment $[71,72,74] 0-50 \%$ at 1 -year $[57,71-73,75,76]$ and $3.9-14 \%$ at 24 months [57, 72]. Recent data report g-tube dependence in patients with late radiation-induced dysphagia ( $\geq 5$-years post-treatment) as a staggering $66 \%$ [63]. With respect to oral intake, $78-86 \%$ pts are able to consume a normal diet without alteration pre-treatment, with the majority of one's diet taken by mouth $[64,77]$. The ability to maintain a normal diet significantly decreases to $10-28.6 \%$ at 3 months, with slow progress toward approx. $50 \%$ at 1 -year post-treatment [64]. Beyond 12 months posttreatment, a trend of declining swallowing functionality emerges, with normal diet rates slipping below $50 \%$ [78].

Relative to minimally invasive surgical techniques, g-tube rates immediately post-surgery range from 13 to $39 \%$ [51, 52, 73], 0 to $19 \%$ at 12 months [51, 53, 73, 79, 80] and $3.4 \%$ at long-term follow-up [52]. Average time to oral intake in early-stage cancers is 2 days [51, 81], and in all-comers, oral intake is achieved by hospital discharge in $70 \%$, and by 2 weeks post-treatment in approximately $80 \%$ [51]. Several studies present impressive results following TORS and/or TLM, with return to normal diet in $53-92 \%$ of patients [80, 81]. Nearly $100 \%$ of patients attain normalcy of diet by 1-year post-surgery [79].

Numerous disease-specific health-related quality of life (HRQOL) and swallowing-related QOL tools have been used to document impact of treatment on the patient's overall well-being. Regardless of the chosen method of assessment, function-related QOL as measured at treatment completion decreases significantly from baseline [68, 74], with significant, albeit modest improvements by 6 months post-treatment, but no further improvement noted at 24 months postCRT [78]. Within the TORS/TLM literature, significant decreases in swallowing-related QOL are noted immediately after treatment completion, with improvement noted between 6 and 12 months post-treatment [51], and some patients experience near-baseline QOL at 1-year post-TORS
[51]. Swallowing-related QOL at 1-year post-TORS/TLM is significantly better in patients compared to QOL in patients who received primary CRT $[51,73,82]$.

Swallowing and dysphagia following treatment of HPV-positive OPSCC

To date, information delineating any relationship that may exist between HPV-positive disease and swallowing physiology or functional outcomes is scarce (Table 2). Bledsoe et al. [83•] retrospectively reviewed outcomes of patients with OPSCC treated with CRT (2006-2011), and correlated oncologic and functional outcomes with HPVpositivity. In this cohort of 121 patients, 97 had HPVpositive disease. Acute grade $\geq 2$ and grade 3 dysphagia appeared with similar frequency in HPV-positive and HPV-negative OPSCC, however, late grade 3 dysphagia occurred more frequently in HPV-negative OPSCC versus HPV-positive OPSCC [83•]. Translating this toxicity into functional impact, the need for enteral feeding in the acute post-treatment period was similar between HPV-negative and HPV-positive disease (58 vs $59 \%$ ), while g-tube rates at 6 months post-treatment were significantly higher in HPV-negative patients (24\%), with no HPV-positive patients continuing to require enteral support [83•]. Indeed, more HPV-positive patients resumed a normal diet (90 vs. $65 \%$ ). It can be hypothesized that fewer late treatment toxicities in HPV-positive patients may reflect differences in patient and tumor characteristics inherent to HPV-positive disease. Rodriguez et al. [84] corroborated these early HPV-related findings in their comparison of two CRT regimens, in which HPV-status was examined. They

Table 2 Literature examining impact of HPV status on swallowing and quality of life

\begin{tabular}{|c|c|c|}
\hline Authors & Primary treatment & $\begin{array}{l}\text { Outcome(s) relative to } \\
\text { HPV status }\end{array}$ \\
\hline $\begin{array}{l}\text { Bledsoe et al. } \\
{\left[83^{\bullet}\right]}\end{array}$ & Chemoradiotherapy & $\begin{array}{l}\text { Dysphagia toxicity } \\
\text { Feeding tube prevalence } \\
\text { Feeding tube duration } \\
\text { Diet level }\end{array}$ \\
\hline $\begin{array}{l}\text { Dobrosotskaya } \\
\text { et al. [85] }\end{array}$ & Chemoradiotherapy & $\begin{array}{l}\text { Feeding tube prevalence } \\
\text { Feeding tube duration }\end{array}$ \\
\hline $\begin{array}{l}\text { Dziegielewski } \\
\text { et al. [88] }\end{array}$ & $\begin{array}{l}\text { Transoral Robotic } \\
\text { Surgery }\end{array}$ & $\begin{array}{l}\text { Health-related quality of } \\
\text { life }\end{array}$ \\
\hline $\begin{array}{l}\text { Maxwell et al. } \\
\text { [87] }\end{array}$ & $\begin{array}{l}\text { Chemoradiotherapy, } \\
\text { Surgery }\end{array}$ & $\begin{array}{l}\text { Health-related quality of } \\
\text { life }\end{array}$ \\
\hline $\begin{array}{l}\text { Rodriguez et al. } \\
\text { [84] }\end{array}$ & Chemoradiotherapy & $\begin{array}{l}\text { Dysphagia toxicity } \\
\text { Feeding tube prevalence } \\
\text { Feeding tube duration }\end{array}$ \\
\hline $\begin{array}{l}\text { Sharma et al. } \\
{\left[86^{\bullet}\right]}\end{array}$ & $\begin{array}{l}\text { Chemoradiotherapy } \\
\text { Surgery }\end{array}$ & $\begin{array}{l}\text { Health-related quality of } \\
\text { life }\end{array}$ \\
\hline
\end{tabular}


document that most of the patients on trial experienced acute grade $\geq 2$ dysphagia, with no correlation to HPV status. Additionally, while feeding tube placement was required during treatment in $64 \%$ of the study population regardless of HPV status, HPV status did predict for shorter duration of feeding tube dependence with median duration of 7 versus 24 weeks in HPV-positive versus HPV-negative disease [84]. It remains possible that treatment intensity could moderate the possible functional benefit noted in the above studies. In the context of a clinical trial examining the treatment efficacy of induction chemotherapy followed by weekly CRT versus standard high-dose cisplatin CRT, g-tube rates at 6 months varied with treatment regimen (26 vs $6 \%$, respectively), but not with HPV status [85].

Sharma et al. [86•] prospectively examined QOL in patients undergoing various treatment approaches for oral cavity and oropharynx cancers relative to HPV status, providing the first glimpse of the impact of HPV on HRQOL in HNSCC. HPV-positive patients demonstrated significantly higher baseline QOL, significantly lower QOL immediately post-treatment, and similar QOL ratings at 1 year post-treatment compared to HPV-negative patients [86•]. The significant interaction between QOL and HPV immediately post-treatment is interesting, and warrants further investigation. Maxwell et al. [87] examined the impact of HPV status and treatment modality on QOL in a similar cohort. Higher overall QOL ratings were reported in patients with HPV-related (p16-positive) versus HPVunrelated OPSCC at baseline, 6 months and 1-to-3 years post-treatment. Additionally, QOL benefit for HPV-related disease was noted in domains of swallowing and speech at $6 \mathrm{M}$ and 1-to-3 years post-treatment [87]. At 2, 6, and 12 months, xerostomia-associated QOL was higher in HPV-negative patients, but this was no longer significant past 1-year post-treatment. Interestingly, they did not report an association between treatment modality and QOL ratings, regardless of HPV status-QOL was more similar between those treated with primary surgery and primary CRT than between patients with HPV-positive versus HPV-negative disease [87]. In contrast, following TORS for OPSCC, Dziegielewski et al. [88] did not find an association between HPV status and QOL ratings, and reported a similar QOL trajectory in HPV-positive and HPV-negative patients. However, the use of disparate QOL measurement tools renders inter-study comparison difficult. Complete understanding of swallowing function and swallowing-related QOL necessitates evaluation of a breadth of parameters-physiologic impairment, functionality, and associated disability-both objectively and subjectively. To date, no investigation of impact of HPV status on treatment outcome in OPSCC has accomplished this task. However, it is encouraging to note data relating HPV status to function are emerging.
Dysphagia management in the era of HPV

Prevention of dysphagia through the implementation of exercise-based therapy and the maintenance of oral intake has become the major focus of swallowing rehabilitation [89]. Engagement in swallowing exercise protocols, both before and during CRT (either as primary or adjuvant treatment) has been associated with improved swallowing physiology, increased functionality of the swallow (i.e., diet levels, g-tube rates), and swallowing-related QOL [58, 8995]. Not all swallow-related exercises are equally effective [96], and the benefit of exercise is mediated by patient adherence to prescribed preventative exercise protocols [72, 89, 97•]. Evidence of further improvements in swallowing performance associated with the addition of transcutaneous neuromuscular electrical stimulation (TNMES) to active exercise regimens is emerging; however, further examination is warranted before TNMES is included as standard of care [98-101]. Use of the swallowing mechanism through oral intake can also predict swallowing-related outcomes (diet level, g-tube rates, swallowing-related QOL) independent of adherence to exercise protocols [89]. Maintenance of safe oral intake may necessitate the use of diet consistency modification (e.g., thickened liquids, pureed solids), bolus manipulation (e.g., smaller volumes, altered sensory properties), and compensatory postural adjustments or voluntary maneuvers (e.g., head turn, super supraglottic swallow), which have been shown to alter bolus flow and overall swallow function $[60,61,102]$. The combination of maintained oral intake and adherence to a swallowing exercise protocol results in best swallowing outcomes for patients receiving CRT [89]. Given the high functional status and short time to resumption of oral intake reported following TORS and/or TLM for OPSCC, dysphagia management may include early post-treatment compensations (i.e., diet modifications, postural adjustments), with emphasis on eating and exercise in the context of adjuvant treatment, although dysphagia management following minimally invasive surgery has not been examined in the literature. In addition, the authors are unaware of any intervention studies that have explicitly examined dysphagia rehabilitation relative to HPV status.

Beyond implementation of rehabilitation practices known to provide functional benefit following CRT for all patients with OPSCC, patient education and counseling of HPV-positive patients is of paramount importance. These younger, healthier, more educated patients, who tend to present with disease in their prime working years, are seeking answers to many questions. It is prudent for clinicians to become knowledgeable with respect to HPV infection, transmission, and vaccination to provide appropriate and helpful information to patients and their families [103, 104••]. In addition, clinicians must educate patients 
receiving CRT (primarily, or as adjuvant therapy) regarding the role of prophylactic/preventative exercises in swallowing recovery. Non-adherence to preventative exercise programs is an independent predictor of g-tube dependence [72, 89], and the most commonly reported reason for non-adherence has been a lack of understanding of the relative importance of these exercises [97•].

\section{Conclusion}

HPV-positive oropharyngeal cancer is a disease on the rise that strikes a younger, healthier, often non-smoking population that experiences improved survival. Swallowing function can be significantly affected by current intensified organ-preserving treatment approaches for OPSCC, with preliminary evidence suggesting that HPV-positive patients experience better swallowing and QOL post-treatment. Moving forward, instrumental assessment in the context of clinical trials will be critical to quantify these functional outcomes in the context of primary radiation and primary surgical treatments. Until further evidence surfaces, we assume that HPV-positive disease will respond similarly to physiologically targeted dysphagia rehabilitation practices as in HPV-negative disease. Dysphagia rehabilitation must include a strong educational focus to highlight the known functional impacts of standard curative treatment(s) and the necessity of maintaining neuromuscular function through exercise and oral intake, and to address questions and concerns inextricably linked to this unique disease.

\section{Compliance with Ethics Guidelines}

Conflict of Interest JA Theurer, J Yoo, and AC Nichols all declare no conflicts of interest.

Human and Animal Rights and Informed Consent All studies by the authors involving animal and/or human subjects were performed after approval by the appropriate institutional review boards. When required, written informed consent was obtained from all participants.

\section{References}

Papers of particular interest, published recently, have been highlighted as:

- Of importance

•- Of major importance

1. Ferlay J, Shin H, Bray F, Forman D, Mathers C, Parkin DM. Estimates of worldwide burden of cancer in 2008: GLOBOCAN 2008. Intl J Cancer. 2010;127:2893-917.

2. Dowthwaite SA, Franklin JH, Palma DA, Fung K, Yoo J, Nichols AC. The role of transoral robotic surgery in the management of oropharyngeal cancer: a review of the literature. ISRN Oncol. 2012;2012:945162.
3. Parsons JT, Mendenhall WM, Stringer SP, et al. Squamous cell carcinoma of the oropharynx: surgery, radiation therapy, or both. Cancer. 2002;94:2967-80.

4. Haigentz J, Missak, Silver CE, Corry J, et al. Current trends in initial management of oropharyngeal cancer: the declining use of open surgery. Eur Arch Otorhinolaryngol. 2009;266:18451855.

5. The Department of Veterans Affairs Laryngeal Cancer Study Group. Induction chemotherapy plus radiation compared with surgery plus radiation in patients with advanced laryngeal cancer. New Engl J Med. 1991;324:1685-960.

6. Staffurth J. Radiotherapy Development Board. A review of the clinical evidence for intensity-modulated radiotherapy. Clin Oncol. 2010;22:643-57.

7. Blanchard P, Hill C, Guihenneuc-Jouyaux C, et al. Mixed treatment comparison meta-analysis of altered fractionated radiotherapy and chemotherapy in head and neck cancer. J Clin Epidemiol. 2011;64:985-92.

8. Bourhis J, Guigay J, Temam S, Pignon JP. Chemo-radiotherapy in head and neck cancer. Ann Oncol. 2006;17(Suppl 10): $\mathrm{x} 39-41$.

9. Pignon J, Maître Al, Maillard E, Bourhis J. Meta-analysis of chemotherapy in head and neck cancer (MACH-NC): an update on 93 randomised trials and 17, 346 patients. Radiother Oncol. 2009;92:4-14.

10. Brizel DM, Albers ME, Fisher SR, et al. Hyperfractionated irradiation with or without concurrent chemotherapy for locally advanced head and neck cancer. New Engl J Med. 1998;338: 1798-804.

11. Eisbruch A, Terrell JE, Wolf GT, et al. Objective assessment of swallowing dysfunction and aspiration after radiation concurrent with chemotherapy for head-and-neck cancer. Int J Radiat Oncol. 2002;53:23-8.

12. Machtay M, Moughan J, Trotti A, et al. Factors associated with severe late toxicity after concurrent chemoradiation for locally advanced head and neck cancer: an RTOG analysis. J Clin Oncol. 2008;26:3582-9.

13. Calais G, Bertrand P, Alfonsi M, et al. Randomized trial of radiation therapy versus concomitant chemotherapy and radiation therapy for advanced-stage oropharynx carcinoma. J Natl Cancer Inst. 1999;91:2081-6.

14. Gillison ML, Symer DE, Shah KV, et al. Evidence for a causal association between human papillomavirus and a subset of head and neck cancers. J Natl Cancer Inst. 2000;92:709-20.

15. Gillison ML. Human papillomavirus-associated head and neck cancer is a distinct epidemiologic, clinical, and molecular entity. Semin Oncol. 2004;31:744-54.

16. Chaturvedi AK, Cozen W, Liu L, et al. Human papillomavirus and rising oropharyngeal cancer incidence in the United States. J Clin Oncol. 2011;29(32):4294-301.

17. Lindquist D, Munck-Wikland E, Dalianis T, et al. Human papillomavirus is a favourable prognostic factor in tonsillar cancer and its oncogenic role is supported by the expression of E6 and E7. Mol Oncol. 2007;1:350-5.

18. Mehanna H, Beech T, Nicholson T, et al. Prevalence of human papillomavirus in oropharyngeal and nonoropharyngeal head and neck cancer-systematic review and meta-analysis of trends by time and region. Head Neck. 2013;35:747-55.

19. Nichols AC, Berk S, Zhou R, et al. The epidemic of human papillomavirus and oropharyngeal cancer in a Canadian population. Curr Oncol. 2013;20:212-9.

20. Chaturvedi AK, Anderson WF, Lortet-Tieulent J, et al. Worldwide trends in incidence rates for oral cavity and oropharyngeal cancers. J Clin Oncol. 2013;31:4550-9.

21. Centers for Disease Control and Prevention. (CDC). Vital signs: current cigarette smoking among adults aged $\geq 18$ years- 
United States, 2005-2010. MMWR Morb Mortal Wkly Rep. 2011;60:1207-11.

22. Davis KC, Farrelly MC, Duke J, et al. Antismoking media campaign and smoking cessation outcomes, New York State, 2003-2009. Prev Chronic Dis. 2012;9:110.

23. de Almeida JR, Genden EM. Robotic surgery for oropharynx cancer: promise, challenges, and future directions. Curr Oncol Rep. 2012;14:148-57.

24. Mork J, Schiller JT, Wang Z, et al. Human papillomavirus infection as a risk factor for squamous-cell carcinoma of the head and neck. New Engl J Med. 2001;344:1125-31.

25. Schwartz SM, Beckmann AM, McDougall JK, et al. Oral cancer risk in relation to sexual history and evidence of human papillomavirus infection. J Natl Cancer Inst. 1998;90:1626-36.

26. Ang KK, Lu C, Kim H, et al. Human papillomavirus and survival of patients with oropharyngeal cancer. New Engl J Med. 2010;363:24-35.

27. D'Souza G, Kreimer AR, Viscidi R, et al. Case-control study of human papillomavirus and oropharyngeal cancer. New Engl J Med. 2007;356:1944-56.

28. Kreimer AR, Clifford GM, Boyle P, Franceschi S. Human papillomavirus types in head and neck squamous cell carcinomas worldwide: a systematic review. Cancer Epidemiol Biomarkers Prev. 2005;14:467-75.

29. Brandsma JL, Abramson AL. Association of papillomavirus with cancers of the head and neck. Arch Otolaryngol Head Neck Surg. 1989;115:621-5.

30. Hollstein M, Sidransky D, Vogelstein B, Harris CC. p53 mutations in human cancers. Science. 1991;253:49-53.

31. Agrawal N, Frederick MJ, Pickering CR, et al. Exome sequencing of head and neck squamous cell carcinoma reveals inactivating mutations in NOTCH1. Science. 2011;333:1154-6.

32. Stransky N, Egloff AM, Tward AD, et al. The mutational landscape of head and neck squamous cell carcinoma. Science. 2011;333:1157-60.

33. Franceschi S, Muñoz N, Bosch XF, Snijders PJ, Walboomers JM. Human papillomavirus and cancers of the upper aerodigestive tract: a review of epidemiological and experimental evidence. Cancer Epidemiol Biomarkers Prev. 1996;5:567-75.

34. Gillison ML, Chaturvedi AK, Lowy DR. HPV prophylactic vaccines and the potential prevention of noncervical cancers in both men and women. Cancer. 2008;113:3036-46.

35. Chung $\mathrm{CH}$, Gillison ML. Human papillomavirus in head and neck cancer: its role in pathogenesis and clinical implications. Clin Cancer Res. 2009;15:6758-62.

36. O'Sullivan B, Huang SH, Perez-Ordonez B, et al. Outcomes of HPV-related oropharyngeal cancer patients treated by radiotherapy alone using altered fractionation. Radiother Oncol. 2012:103:49-56.

37. Gillison ML, Broutian T, Pickard RKL, et al. Prevalence of oral HPV infection in the United States, 2009-2010. JAMA. 2012:307:693-703.

38. Lindel K, Beer KT, Laissue J, Greiner RH, Aebersold DM. Human papillomavirus positive squamous cell carcinoma of the oropharynx: a radiosensitive subgroup of head and neck carcinoma. Cancer. 2001;92:805-13.

39. Fakhry C, Westra WH, Li S, et al. Improved survival of patients with human papillomavirus-positive head and neck squamous cell carcinoma in a prospective clinical trial. J Natl Cancer Inst. 2008;100:261-9.

40. Kumar B, Cordell KG, Lee JS, et al. EGFR, p16, HPV titer, Bcl-xL and 553 , sex, and smoking as indicators of response to therapy and survival in oropharyngeal cancer. J Clin Oncol. 2008;26:3128-37.

41. Li W, Rose BR, Thompson $\mathrm{CH}$, et al. Human papillomavirus positivity predicts favourable outcome for squamous carcinoma of the tonsil. Int J Cancer. 2003;106:553-8.
42. Licitra L, Squadrelli M, Quattrone P, et al. High-risk human papillomavirus affects prognosis in patients with surgically treated oropharyngeal squamous cell carcinoma. J Clin Oncol. 2006;24:5630-6.

43. Ragin CCR, Taioli E. Survival of squamous cell carcinoma of the head and neck in relation to human papillomavirus infection: review and meta-analysis. Int J Cancer. 2007;121:1813-20.

44. Huang SH, Weinreb I, Hope A, et al. Atypical clinical behavior of p16-confirmed HPV-related oropharyngeal squamous cell carcinoma treated with radical radiotherapy. Int J Radiat Oncol. 2012;82:276-83.

45. Broglie MA, Soltermann A, Rohrbach D, et al. Impact of p16, p53, smoking, and alcohol on survival in patients with oropharyngeal squamous cell carcinoma treated with primary intensitymodulated chemoradiation. Head Neck. 2013;35:1698-706.

46. Nguyen NP, Smith HJ, Sallah S. Evaluation and management of swallowing dysfunction following chemoradiation for head and neck cancer. Curr Opin Otolaryngol Head Neck Surg. 2007;15: 130-3.

47. O'Sullivan B, Huang SH, Siu LL, et al. Deintensification candidate subgroups in human papillomavirus-related oropharyngeal cancer according to minimal risk of distant metastasis. J Clin Oncol. 2013;31:543-50.

48. Ferris RL, Jaffee EM, Ferrone S. Tumor antigen-targeted, monoclonal antibody-based immunotherapy: clinical response, cellular immunity, and immunoescape. J Clin Oncol. 2010;28: 4390-9.

49. Bonner JA, Jassem J, Ove R, et al. Radiotherapy plus cetuximab for squamous-cell carcinoma of the head and neck. New Engl J Med. 2006;354:567-78.

50. ClinicalTrials.gov [homepage on the Internet]. RTOG 1016: Phase III trial of radiotherapy plus cetuximab versus chemoradiotherapy in HPV-associated oropharynx cancer. Available from: http://clinicaltrials.gov/ct2/show/NCT01302834?term= RTOG+1016\&rank=1. Accessed 15 July 2014.

51. Hutcheson KA, Holsinger FC, Kupferman ME, Lewin JS. Functional outcomes after TORS for oropharyngeal cancer: a systematic review. Eur Arch Otorhinolaryngol. 2014. Accepted Article. doi:10.1007/s00405.

52. Rich JT, Milov S, Lewis JS Jr, et al. Transoral laser microsurgery $(\mathrm{TLM}) \pm$ adjuvant therapy for advanced stage oropharyngeal cancer: outcomes and prognostic factors. Laryngoscope. 2009;119:1709-19.

53. Haughey BH, Hinni ML, Salassa JR, et al. Transoral laser microsurgery as primary treatment for advanced-stage oropharyngeal cancer: a United States multicenter study. Head Neck. 2011;33:1683-94.

54. Nichols AC, Kuruvilla S, Chen J, et al. Early-stage squamous cell carcinoma of the oropharynx: radiotherapy versus trans-oral robotic surgery (ORATOR) - study protocol for a randomized phase II trial. BMC Cancer. 2013;13:133.

55. ClinicalTrials.gov [homepage on the Internet]. RTOG 1221: Randomized phase II trial of transoral endoscopic head and neck surgery followed by risk-based IMRT and weekly cisplatin versus IMRT and weekly cisplatin for HPV negative oropharynx cancer. Available from: http://clinicaltrials.gov/ct2/show/ NCT01953952?term=RTOG+1221\&rank=1. Accessed 15 July, 2014.

56. •• Wall LR, Ward EC, Cartmill B, Hill AJ. Physiological changes to the swallowing mechanism following (chemo)radiotherapy for head and neck cancer: a systematic review. Dysphagia. 2013;28:481-493. This is a comprehensive systematic review of the physiologic swallowing impairments encountered following chemoradiotherapy. It supports evidence-based practice by identifying the most salient physiologic targets for swallowing rehabilitative exercises. 
57. Hutcheson KA, Lewin JS. Functional outcomes after chemoradiotherapy of laryngeal and pharyngeal cancers. Curr Oncol Rep. 2012;14:158-65.

58. Roe JWG, Ashforth KM. Prophylactic swallowing exercises for patients receiving radiotherapy for head and neck cancer. Curr Opin Otolaryngol Head Neck Surg. 2011;19:144-9.

59. Mittal BB, Pauloski BR, Haraf DJ, et al. Swallowing dysfunction-preventative and rehabilitation strategies in patients with head-and-neck cancers treated with surgery, radiotherapy, and chemotherapy: a critical review. Int J Radiat Oncol. 2003;57: 1219-30.

60. Pauloski BR, Rademaker AW, Logemann JA, Discekici-Harris M, Mittal BB. Comparison of swallowing function after IMRT and conventional radiotherapy for head and neck cancer. Head Neck. 2014. Accepted Article. doi: 10.1002/hed.23796.

61. Rogus-Pulia NM, Pierce M, Mittal BB, Zecker SG, Logemann J. Bolus effects on patient awareness of swallowing difficulty and swallow physiology following chemoradiation for head and neck cancer. Head Neck. 2014. Accepted Article. doi:10.1002/ hed. 23720

62. Bleier BS, Levine MS, Mick R, et al. Dysphagia after chemoradiation: analysis by modified barium swallow. Ann Oto Rhinol Laryngol. 2007;116:837-41.

63. Hutcheson KA, Lewin JS, Barringer DA, et al. Late dysphagia after radiotherapy-based treatment of head and neck cancer. Cancer. 2012;118:5793-9.

64. Logemann JA, Mittal B, Pauloski BR, et al. Swallowing disorders in the first year after radiation and chemoradiation. Head Neck. 2008;30:148-58.

65. National Cancer Institute. Common Terminology Criteria for Adverse Events v.4.0 (CTCAE). Available at http://ctep.cancer. gov/protocolDevelopment/electronic_applications/ctc.htm. Accessed 02 July 2014.

66. Trotti A. Toxicity in head and neck cancer: a review of trends and issues. Int J Radiat Oncol. 2000;47:1-12.

67. Al-Mamgani A, van Rooij P, Verduijn GM, et al. The impact of treatment modality and radiation technique on outcomes and toxicity of patients with locally advanced oropharyngeal cancer. Laryngoscope. 2013;123:386-93.

68. Al-Mamgani A, van Rooij P, Sewnaik A, et al. Brachytherapy or stereotactic body radiotherapy boost for early-stage oropharyngeal cancer: comparable outcomes of two different approaches. Oral Oncol. 2013;49:1018-24.

69. Feng M, Feng FY, Kim HM, et al. Intensity-modulated radiotherapy of head and neck cancer aiming to reduce dysphagia: early dose-effect relationships for the swallowing structures. Int J Radiat Oncol. 2007;68:1289-98.

70. Hunter K, Eisbruch A, Schipper M, et al. Toxicities affecting quality of life after chemo-IMRT of oropharyngeal cancer: prospective study of patient-reported, observer-rated, and objective outcomes. Int J Radiat Oncol. 2013;85:935-40.

71. McBride SM, Parambi RJ, Jang JW, Goldsmith T, Busse PM, Chan AW. Intensity-modulated versus conventional radiation therapy for oropharyngeal carcinoma: long-term dysphagia and tumor control outcomes. Head Neck. 2014;36:492-8.

72. Bhayani MK, Hutcheson KA, Barringer DA, et al. Gastrostomy tube placement in patients with oropharyngeal carcinoma treated with radiotherapy or chemoradiotherapy: Factors affecting placement and dependence. Head Neck. 2013;35:1634-40.

73. Chen AM, Daly ME, Luu Q, Donald PJ, Farwell DG. Comparison of functional outcomes and quality of life between transoral surgery and definitive chemoradiotherapy for oropharyngeal cancer: functional outcomes and oropharyngeal Cancer. Head Neck. 2014. Accepted Article. doi:10.1002/hed.23610.

74. Cartmill B, Cornwell P, Ward E, Davidson W, Porceddu S. Swallowing, nutrition and patient-rated functional outcomes at
6 months following two non-surgical treatments for T1-T3 oropharyngeal cancer. Support Care Cancer. 2012;20:2073-81.

75. Bhide SA, Newbold KL, Harrington KJ, Nutting CM. Clinical evaluation of intensity-modulated radiotherapy for head and neck cancers. Br J Radiol. 2012;85:487-94.

76. Givens DJ, Karnell LH, Gupta AK, et al. Adverse events associated With concurrent chemoradiation therapy in patients with head and neck cancer. Arch Otolaryngol Head Neck Surg. 2009;135:1209-17.

77. Cartmill B, Cornwell P, Ward E, Davidson W, Porceddu S. A prospective investigation of swallowing, nutrition, and patientrated functional impact following altered fractionation radiotherapy with concomitant boost for oropharyngeal cancer. Dysphagia. 2012;27:32-45.

78. Cartmill B, Cornwell P, Ward E, Davidson W, Porceddu S. Long-term functional outcomes and patient perspective following altered fractionation radiotherapy with concomitant boost for oropharyngeal cancer. Dysphagia. 2012;27:481-90.

79. Moore EJ, Olsen KD, Olsen SM, et al. Long-term functional and oncologic results of transoral robotic surgery for oropharyngeal squamous cell carcinoma. Mayo Clin Proc. 2012;87:219-25.

80. Steiner W, Fierek O, Ambrosch P, Hommerich CP, Kron M. Transoral laser microsurgery for squamous cell carcinoma of the base of the tongue. Arch Otolaryngol Head Neck Surg. 2003;129: 36-43.

81. Aubry K, Yachine M, Perez A, et al. Transoral robotic surgery for head and neck cancer: a series of 17 cases. Eur Ann Otorhinolaryngol. 2011;128:290-6.

82. O'Hara J, Cosway B, Muirhead C, Leonard N, Goff D, Patterson J. Transoral laser microsurgery \pm adjuvant therapy versus chemoradiotherapy for stage III and IVA oropharyngeal squamous cell cancer: A preliminary comparison of early swallowing outcomes. Head Neck. 2014; Accepted Article. doi:10.1002/hed.23790.

83. - Bledsoe T, Koyfman S, Noble A, et al. Oropharyngeal squamous cell carcinoma with known human papillomavirus status treated with definitive chemoradiotherapy: patterns of failure and toxicity outcomes. Radiat Oncol. 2013;8:174-174. This study provides preliminary evidence to support the hypothesis that patients with HPV-positive disease experience better functional outcomes, in addition to superior oncologic outcomes, compared to patients with HPV-negative disease.

84. Rodriguez CP, Adelstein DJ, Rybicki LA, et al. A randomized phase III study of two cisplatin based chemoradiation (CCRT) regimens in locally advanced head and neck squamous cell carcinoma (LAHNSCC): the impact of changing disease epidemiology on contemporary trial design. Head Neck. 2014. Accepted Article. doi: 10.1002/hed.23794.

85. Dobrosotskaya IY, Teknos T, Chepeha DB, et al. Weekly chemotherapy with radiation versus high-dose cisplatin with radiation as organ preservation for patients with HPV-positive and HPV-negative locally advanced squamous cell carcinoma of the oropharynx. Head Neck. 2014;36:617-23.

86. - Sharma A, Chen C, Méndez E, et al. Human papillomaviruspositive oral cavity and oropharyngeal cancer patients do not have better quality-of-life trajectories. Otolaryngol Head Neck Surg. 2012;146:739-745. This prospective study examined quality of life following primary cancer treatment, and provides first evidence that although patients with HPV-positive disease experience better function and prognosis, their post-treatment quality of life trajectory is similar to patients with HPV-negative disease.

87. Maxwell JH, Mehta V, Wang H, et al. Quality of life in head and neck cancer patients: Impact of HPV and primary treatment modality. Laryngoscope. 2014;124:1592-7.

88. Dziegielewski PT, Teknos TN, Durmus K, et al. Transoral robotic surgery for oropharyngeal cancer: long-term quality of 
life and functional outcomes. JAMA Otolaryngol Head Neck Surg. 2013;139:1099-108.

89. Hutcheson KA, Bhayani MK, Beadle BM, et al. Eat and exercise during radiotherapy or chemoradiotherapy for pharyngeal cancers: use it or lose it. JAMA Otolaryngol Head Neck Surg. 2013;139:1127-34.

90. Carroll WR, Locher JL, Canon CL, Bohannon IA, McColloch NL, Magnuson JS. Pretreatment swallowing exercises improve swallow function after chemoradiation. Laryngoscope. 2008;118:39-43.

91. Kulbersh BD, Rosenthal EL, McGrew BM, et al. Pretreatment, preoperative swallowing exercises may improve dysphagia quality of life. Laryngoscope. 2006;116:883-6.

92. Carnaby-Mann G, Crary MA, Schmalfuss I, Amdur R. "Pharyngocise": Randomized controlled trial of preventative exercises to maintain muscle structure and swallowing function during head-and-neck chemoradiotherapy. Int J Radiat Oncol. 2012;83:210-9.

93. Crary MA, Carnaby GD, LaGorio LA, Carvajal PJ. Functional and physiological outcomes from an exercise-based dysphagia therapy: a pilot investigation of the McNeill Dysphagia Therapy Program. Arch Phys Med Rehab. 2012;93:1173-8.

94. Kotz T, Federman AD, Kao J, et al. Prophylactic swallowing exercises in patients with head and neck cancer undergoing chemoradiation: a randomized trial. Arch Otolaryngol Head Neck Surg. 2012;138:376-82.

95. van der Molen L, van Rossum MA, Burkhead LM, Smeele LE, Rasch CRN, Hilgers FJM. A randomized preventive rehabilitation trial in advanced head and neck cancer patients treated with chemoradiotherapy: feasibility, compliance, and short-term effects. Dysphagia. 2011;26:155-70.

96. Lazarus CL, Smith B, Sanfilippo N, et al. Effects of exercise on swallowing and tongue strength in patients with oral and oropharyngeal cancer treated with primary radiotherapy with or without chemotherapy. Int J Oral Max Surg. 2014;43:523-30.
97. - Shinn EH, Hutcheson KA, Barringer D, et al. Adherence to preventive exercises and self-reported swallowing outcomes in post-radiation head and neck cancer patients. Head Neck. 2013;35:1707-1712. This prospective study emphasizes the rehabilitative importance of swallowing therapeutic exercise protocols, and provides insight into factors that may influence patient adherence. These factors may allow clinicians to improve patient adherence, and ultimately, patient outcome.

98. Carnaby-Mann GD, Crary MA. Examining the evidence on neuromuscular electrical stimulation for swallowing: a metaanalysis. Arch Otolaryngol Head Neck Surg. 2007;133:564-71.

99. Carnaby-Mann GD, Crary MA. Adjunctive neuromuscular electrical stimulation for treatment-refractory dysphagia. Ann Otol Rhinol Laryngol. 2008;117:279-87.

100. Ryu JS, Kang JY, Park JY, et al. The effect of electrical stimulation therapy on dysphagia following treatment for head and neck cancer. Oral Oncol. 2009;45:665-8.

101. Bhatt A, Goodwin N, Bhatt G, et al. Impact of transcutaneous neuromuscular electrical stimulation for dysphagia in head-andneck cancer patients treated with definitive chemoradiation. Int J Radiat Oncol. 2012;84:S62.

102. Pauloski BR, Logemann JA, Rademaker AW, et al. Effects of enhanced bolus flavors on oropharyngeal swallow in patients treated for head and neck cancer. Head Neck. 2013;35:1124-31.

103. Baxi SS, Shuman AG, Corner GW, et al. Sharing a diagnosis of HPV-related head and neck cancer: the emotions, the confusion, and what patients want to know. Head Neck. 2013;35:1534-41.

104. • Fakhry C, D'Souza G. Discussing the diagnosis of HPVOSCC: common questions and answers. Oral Oncol. 2013;49:863-871. This article addresses questions raised commonly by patients following receipt of a diagnosis of $\mathrm{HPV}$ related cancer. A sample brochure that clinicians may use for patient education is provided. 